\title{
Pulsed Supersonic Beams from High Pressure Source: Simulation Results and Experimental Measurements
}

\author{
U. Even \\ Sackler School of Chemistry, Tel Aviv University, 69978 Tel Aviv, Israel \\ Correspondence should be addressed to U. Even; even@post.tau.ac.il \\ Received 2 April 2014; Revised 5 June 2014; Accepted 26 June 2014; Published 3 August 2014 \\ Academic Editor: Zhengcheng Zhang \\ Copyright (C) 2014 U. Even. This is an open access article distributed under the Creative Commons Attribution License, which \\ permits unrestricted use, distribution, and reproduction in any medium, provided the original work is properly cited. \\ Pulsed beams, originating from a high pressure, fast acting valve equipped with a shaped nozzle, can now be generated at high \\ repetition rates and with moderate vacuum pumping speeds. The high intensity beams are discussed, together with the skimmer \\ requirements that must be met in order to propagate the skimmed beams in a high-vacuum environment without significant \\ disruption of the beam or substantial increases in beam temperature.
}

\section{Introduction}

Supersonic beams have proven to be an essential source of information on molecular properties and collision processes. The early stage of their development relied on the production of continuous beams and was limited by available pumping capacity. Beam propagation was limited by collisions with background gases, usually to a distance of only a few $\mathrm{mm}$ before the beam was dispersed and attenuated. Much effort was required to ensure that the jet was interrogated before it was degraded, and building such a supersonic beam instrument became an art [1-5].

The introduction of pulsed supersonic beams solved some of the problems of CW (continuous wave) machines and is widely spread today, covering too many research areas to enumerate concisely. Since its early years it was evident that using pulsed beams enables the generation of more intense and colder beams at reduced pumping capacity [14]. The short pulse length $(<50 \mu \mathrm{sec}$.) enables us to disregard interaction with the walls of the vacuum system or residual gas. In fact only interactions within a radius of $20 \mathrm{~mm}$ from the beam are relevant to these time scales, and the pumps have all the time between pulses to reduce the background pressure to negligible values.

Many designs of pulsed beam sources are based on various actuating mechanisms (mechanical [6], Lorenz force [79], Piezo driver [10, 11], or electromagnetic [12-17]). Several years ago we introduced a shaped nozzle, which combined with a high pressure fast-acting pulsed valve enabled us to reach high beam intensities and lower jet temperatures than what was previously available $[18,19]$. The development of this valve and its operating characteristics are described here, along with the changes in skimmer design that are required in order to make use of the higher on-axis beam intensities. A comprehensive review of our pulsed valve was recently published [20]. Simulations of gas flow [21] as well as measurements of actual beam parameters are presented. Previous designs of molecular beam skimmers [5, 20, 22-24] as originally developed for the earlier low-intensity continuous beams cannot be copied for high intensity beams because skimmer interference (also known as skimmer clogging) is simply too severe with the more intense pulsed beams that are now available. In this paper, we describe recent results on nozzle design (Section 2), pulsed valve design (Section 3), molecular beam properties (Section 4), skimmer design (Section 5), and conclusions (Section 6). These topics are discussed in the designated sections, Section 2-Section 6.

\section{Nozzle Design and Testing}

The simplest nozzle is a circular hole in a thin plate also known as a sonic nozzle. The expansion from this simple geometry can be calculated analytically [3], but we choose 

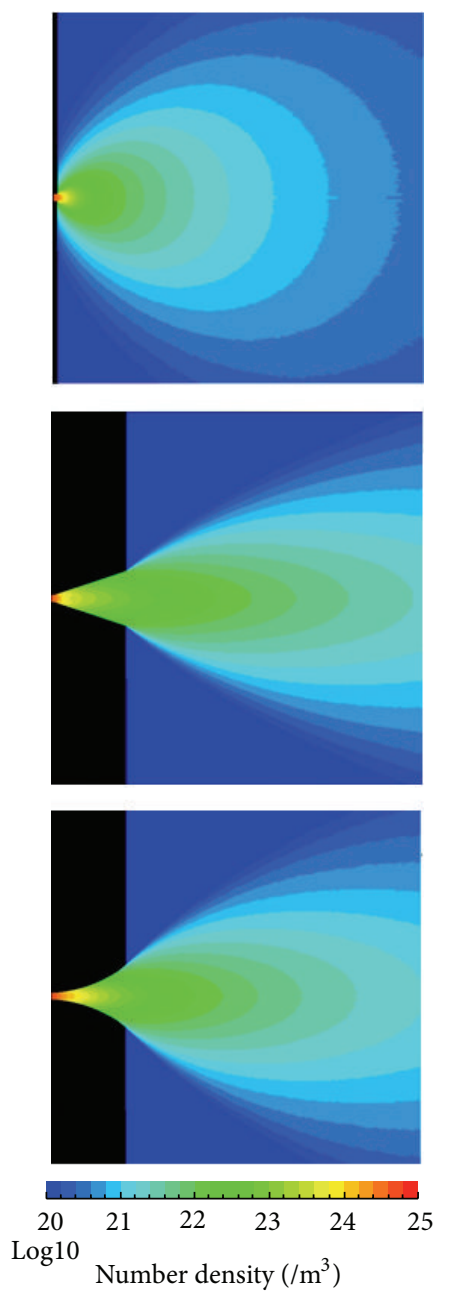

Figure 1: Number density contour for three nozzles.

to show a simulation of this axisymmetric flow because this allows for more direct comparisons to the results for more complicated nozzle shapes. We use the freely available DSMC program [25], run on a modern personal computer for several hours for each run. The simulation run contains $\sim 1$ million atoms and becomes too long if we use a stagnation pressure larger than 0.3 bars expanding into a vacuum. The mean free path of the flow in the nozzle itself (and up to 50 nozzle diameters) is much smaller than the dimensions involved, leading us to believe that the results at this reduced pressure simulations ( 0.3 bars) can also represent the flow at the higher operating pressures (several tens of bars). No clustering is included in the simulation here. The simulation was run until no further changes are seen; that is, a steady state is achieved in the flow (50 ms of flow time). The simulation results are then compared to experimental results to test this assumption. We present results for the beam intensity (i.e., atomic number density) and Mach number contours for three nozzles: (I) sonic nozzle, (II) conical nozzle of 40 degrees full angle, and (III) parabolic nozzle shape. All nozzles have the same inlet diameters $(0.2 \mathrm{~mm})$. The last two nozzles have a length of $2 \mathrm{~mm}$ (10 nozzle diameters). The computation
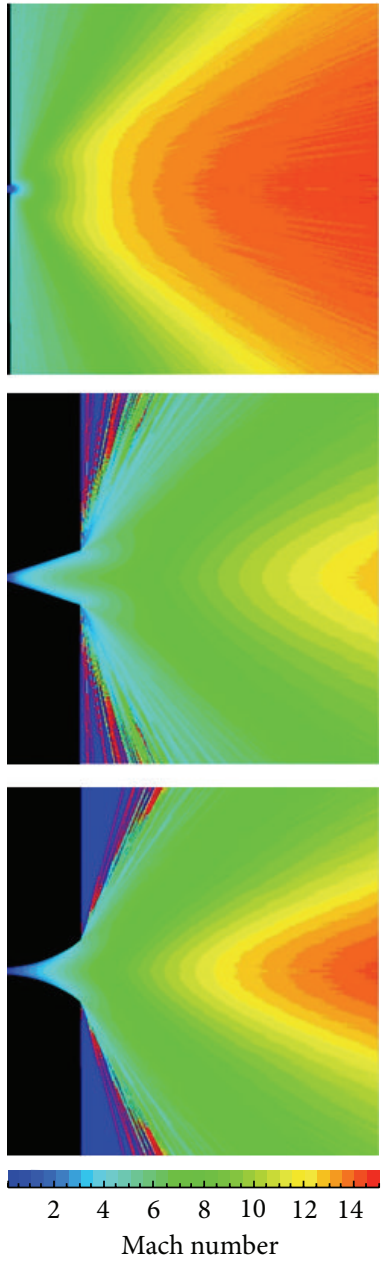

FIGURE 2: Mach number contours for three nozzles.

extends to $10 \mathrm{~mm}$ distance from the nozzle (50 nozzle diameters). Figures 1 and 2 show the simulation results. Since the computations are performed at low stagnation pressures, we extrapolate that actual beam density in our experiments will be 100 times higher (at the applied experimental pressure of 30 bars). A central issue in the computation is how to model the interaction of individual atoms colliding with the nozzle surface. This is also known as the energy accommodation coefficients problem. Not much is known for our nozzle surface (polished stainless steel or more recently polished ruby). We chose a perpendicular accommodation coefficient of 0.4 and a tangential accommodation coefficient of 0.4 [2629] in the simulations to simulate helium. These coefficients determine the boundary layer thickness in the flow (between the flowing gas and the stationary nozzle surface). We estimate that at the applied high pressures in our experiment this boundary layer is less than 10 micrometers thick.

Figure 1 displays the expected $\cos (\theta)$ angular dependence [30] for the sonic nozzle resulting in a wide beam with a rapid density fall-off and rapid cooling with distance (Mach number in Figure 2). The other two shaped nozzles show a slower expansion rate as the gas jet is confined by the nozzles walls. 


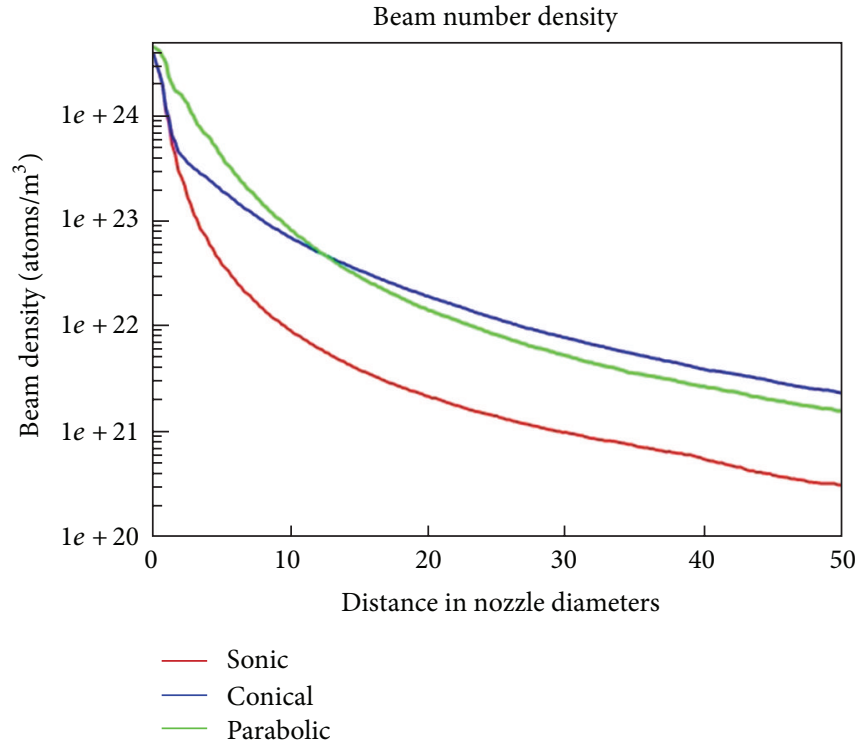

(a)

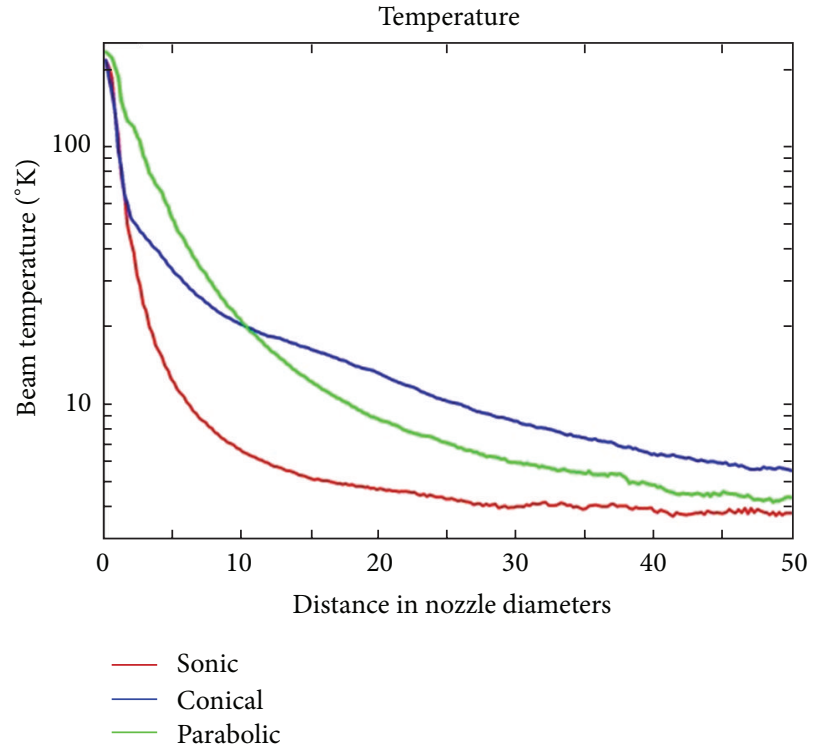

(b)

FIGURE 3: Number density and temperature as a function of the distance from the nozzle.

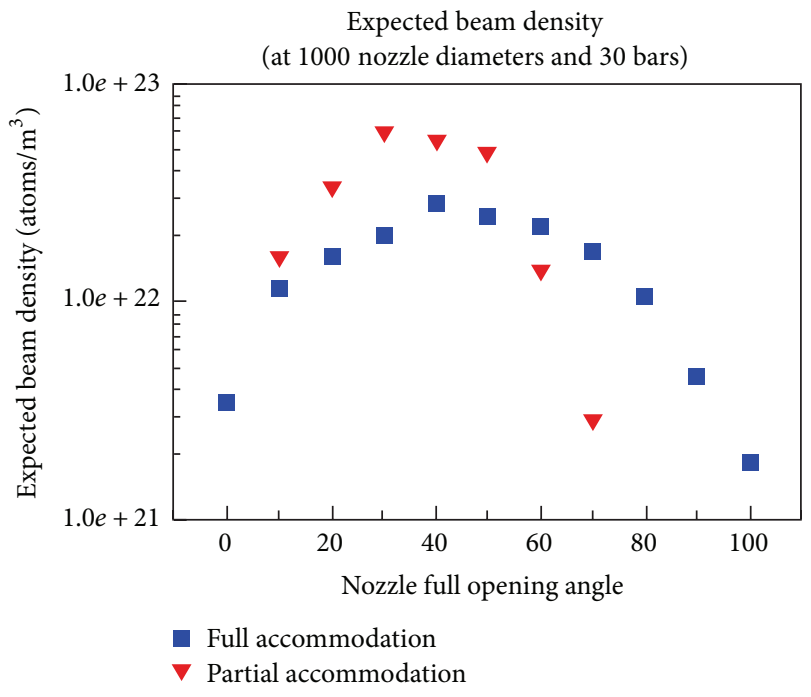

(a)

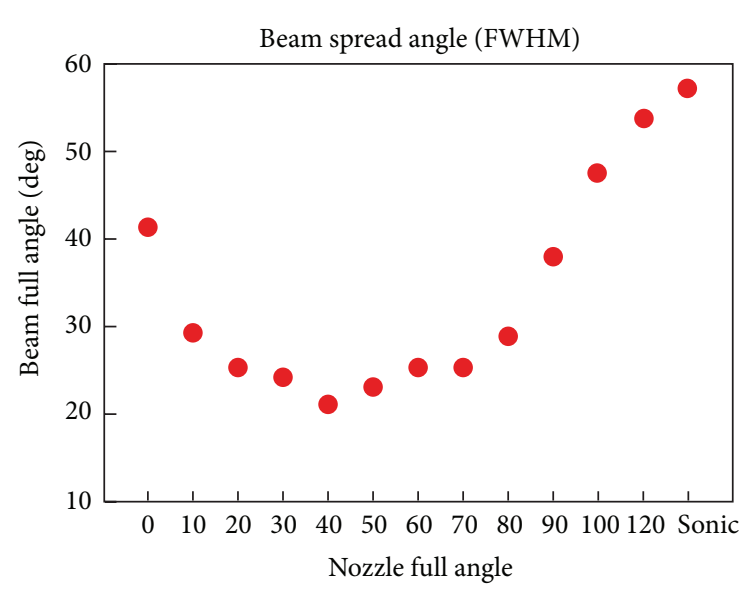

(b)

FIGURE 4: Beam density and angular spread (FWHM) as a function of the conical nozzle opening angle. Two accommodation models are shown in (a) (energy accommodation is 1 or 0.4 ); we calculated the expected beam density at a distance of 500 nozzle diameters (100 mm.) and a stagnation pressure of 30 bars. Admittedly this is a wild extrapolation of the simulated results which were performed at 0.3 bars only.

Figure 3 depicts the fall in number density and temperature with the distance from the nozzle. Both cone nozzle and parabolic nozzle show a slower expansion rate resulting in higher beam number density (i.e., beam intensity) at a given distance from the nozzle. This slower expansion results in a many more collisions before the beam density becomes too low (the so called sudden freeze model [5, 31-36]), enabling more efficient cooling due to two-body collisions, as well as encouraging cluster growth via threebody collisions. One can conclude from these simulations that a conical or parabolic nozzle is much better than a sonic nozzle for obtaining high on-axis beam intensities. Either design provides an improvement of about a factor of five in beam intensity compared to the sonic nozzle. The parabolic nozzle is advantageous for the growth of weakly bound clusters because it avoids the prolonged period in which the expanding gas is held at $20-30 \mathrm{~K}$ that is found with the conical nozzle design.

Figure 4 shows the simulated on-axis beam atomic number density as a function of the opening angle of the cone 
nozzle. As can be seen, $40^{\circ}-50^{\circ}$ cones represent an optimum choice of opening angle. This is intuitively reasonable, as there is no difference between the limiting case of a low opening angle approaching $0^{\circ}$ and a large opening angle approaching $180^{\circ}$.

We can compare these simulation results with experimental measurement of beam intensity and angular dependence in Figure 5.

These simulation results are compared to measurements of actual beam intensity as a function of angle in Figure 5, where results for sonic and $40^{\circ}$ conical nozzles are presented for the same expansion conditions. The beam intensity was measured with a fast ion gauge as the nozzle was rotated. The conical nozzle produced an on-axis beam intensity that was more than a factor of 10 greater than the sonic nozzle. The greater increase in on-axis beam intensity compared to simulation results is probably due to the higher pressure used in the experiment, as compared to the simulation, which resulted in a thinner boundary layer near the nozzle wall.

Verification of the production of narrow, collimated beams from a shaped nozzle is demonstrated visually in Figure 6. Here a neon beam, excited by an electric discharge inside the conical nozzle [37], is photographed. The emerging excited neon atoms emit in the red, showing that the expansion diverges at an angle of approximately $10^{\circ}$ (FWHM). The angular distribution of the emitting neon atoms is consistent with the measured angular distribution presented in Figure 5 and is a bit narrower than the simulated beam density of Figure 1.

\section{Valve Design}

The design of the pulsed valve is described below. It is actuated by a short $(\sim 30 \mu \mathrm{s})$, high current ( 20 A), and low voltage $(\sim 30 \mathrm{~V})$ pulse. This creates a strong pulsed magnetic field ( 3 Tesla) that imparts momentum to the magnetic alloy stainless steel plunger. The coil design was simulated by solving the Maxwell equations using the properties of the actual materials. The resulting spatial distribution of the magnetic field is displayed in Figure 7.

The magnetic field was also measured using a small Faraday rotation crystal $[38,39]$ inserted inside the coil. The magnetic fields generated by the coil interact with the plunger to create an actuating force of about $10 \mathrm{~N}$. The air gap and therefore the free movement of the plunger are limited to $50 \mu \mathrm{m}$ only. The materials placed in the coil center are subjected to high fields and can easily reach deep saturation. In fact not enough data can be found in the literature to accurately predict material behavior under these extreme fields. However, the simulation does predict the measured response time quite accurately (opening time of $\sim 20 \mu \mathrm{s}$.).

The assembled valve designed for room temperature operation is shown in Figure 9. Some modifications in the construction materials allow the valve to operate without adjustments from $10 \mathrm{~K}$ to $500 \mathrm{~K}$.

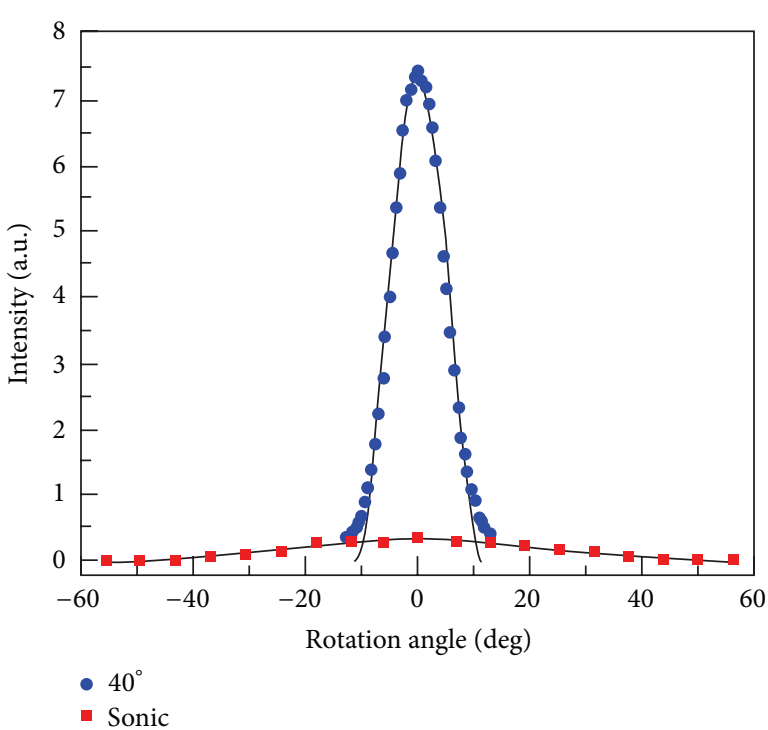

FIGURE 5: Experimental measurement of angular distribution of molecular beam intensity for sonic and $40^{\circ}$ conical nozzles. Experimental conditions: 100 bars helium at $300 \mathrm{~K}$. Nozzle length is 10 nozzle diameters. Beam intensity was measured by a fast ionization gauge [18].

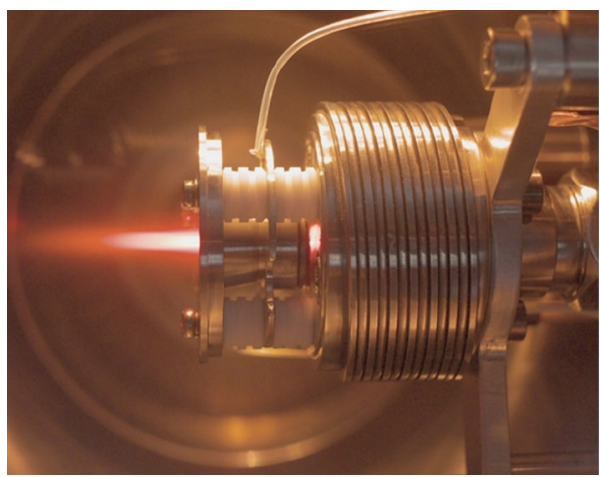

FIGURE 6: Low divergence of an excited neon beam from a conical nozzle.

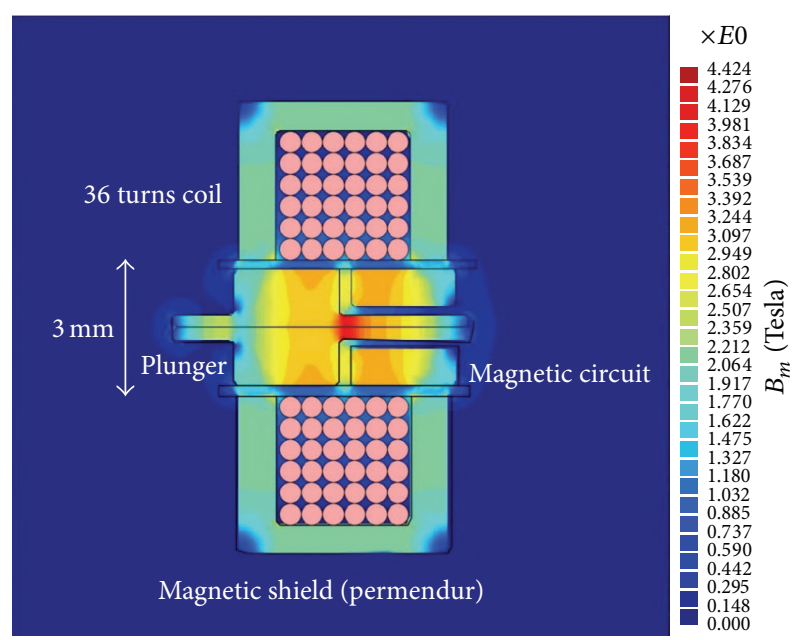

FIGURE 7: Coil and plunger forming the actuating magnetic circuit [14]. 


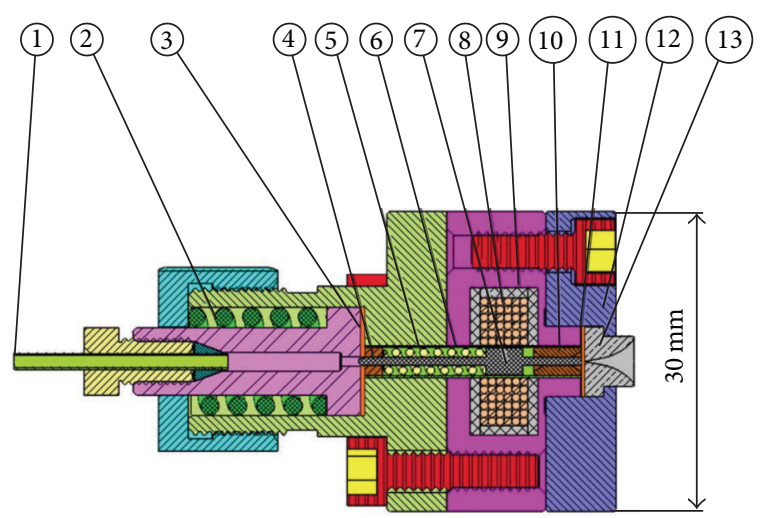

FIgURE 8: Assembled room temperature Even-Lavie valve [14].

From Figure 8 we have the following pulsed valve components:

(1) stainless gas inlet tube $\left(1 / 16^{\prime \prime}\right)$ gas feed;

(2) tightening spring $(180 \mathrm{~N})$ and pressure relief valve;

(3) Kapton foil gasket seals (rear, $0.125 \mathrm{~mm}$. thick);

(4) ceramic (Zirconia or Ruby) rear guiding precision ferrule;

(5) return spring (stainless alloy);

(6) thin walled pressure vessel (Inconel or Zirconia ceramic);

(7) reciprocating plunger (magnetic stainless steel alloy);

(8) Kapton insulated copper coil;

(9) permendur magnetic shield and field concentrator;

(10) ceramic (Zirconia or Ruby) front guiding precision ferrule;

(11) Kapton foil gasket seal (front, $0.125 \mathrm{~mm}$. thick);

(12) front flange and valve body (copper or stainless);

(13) conical (or parabolic) shape expansion nozzle (Zirconia ceramic or hardened stainless steel).

Some basic operating specifications of the valve are given below:

(1) spring pressure that maintains sealing under full temperature range $10-500 \mathrm{~K}$;

(2) pressure range 0-100 bars;

(3) corrosion resistant materials (stainless steel, ceramics, and Kapton);

(4) generated gas pulse width 20-30 $\mu$ s;

(5) repetition rate $0-600 \mathrm{~Hz}$;

(6) energy consumption $6 \mathrm{~mJ}$ per pulse.

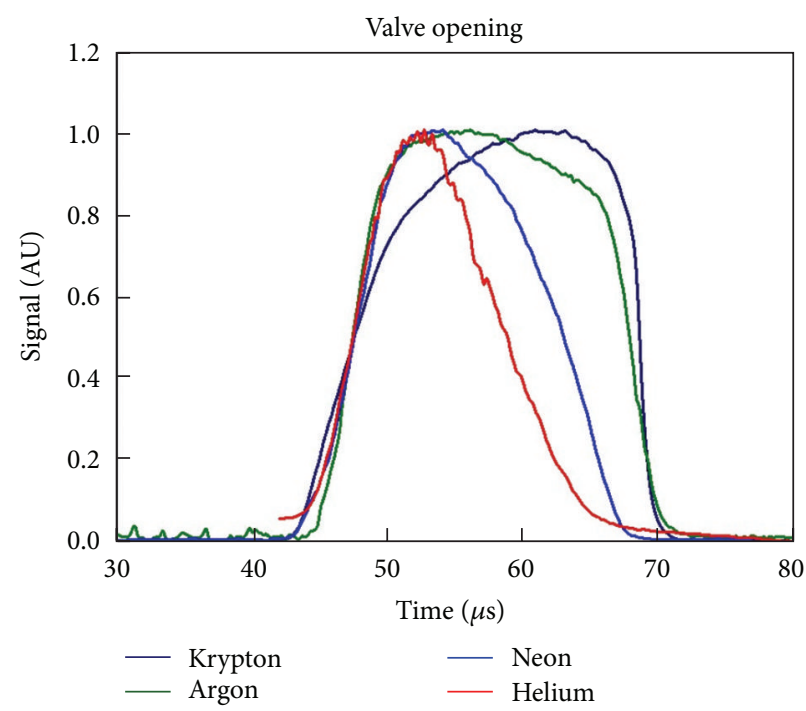

FIGURE 9: Gas pulse duration at the nozzle exit.

\section{Beam Properties Generated by the Even-Lavie Valve}

The time response, intensity, and the speed ratio of the supersonic jets, generated by this valve, were measured [40, 41]. The operating gases and stagnation conditions (pressure and temperature) were varied.

Time in Figure 9 is measured from the initiating current pulse rise. The gas pulse duration is not determined solely by the excitation current pulse duration. The plunger movement is determined by the initial momentum gained from the magnetic field pulse and the time to recoil from the end of its travel gap. There is a built in delay of $\sim 45 \mu \mathrm{s}$, before the gas appears at the nozzle exit. Driving the valve with higher currents (or larger current pulse width) can eventually cause the plunger to bounce more than once, creating multiple pulses of gas. Note the effect of gas viscosity (and its molecular velocity) on the gas pulse duration and its delay. The heavier gases appear later and have longer pulse duration.

Once the gas pulse is formed (with a pulse duration of $20 \mu \mathrm{s}$ corresponding to an axial length of $30 \mathrm{~mm}$ for helium at $300 \mathrm{~K}$ ), it propagates unimpeded if the background pressure is sufficiently low. At pressures of 10-4 mbar, however, there is already severe beam attenuation over a distance of several $\mathrm{cm}$. To experimentally investigate the gas pulse, we have monitored it using a fast ion gauge. One must remember that at the beginning and the end of the plunger movement the valve is not fully opened, so the beam properties (such as pressure and speed) change during the gas pulse. To measure the properties of the fully developed expansion more accurately, it would be better to select only the central part of the pulse (say, at the peak opening of the valve) and measure the properties of the expanding gas in that part of the pulse. One way that this could be accomplished would be to use a short duration pulsed and focused electron beam, fired at the appropriate time to excite a narrow group of atoms in the central portion of the pulse. A fraction of the atoms will be 


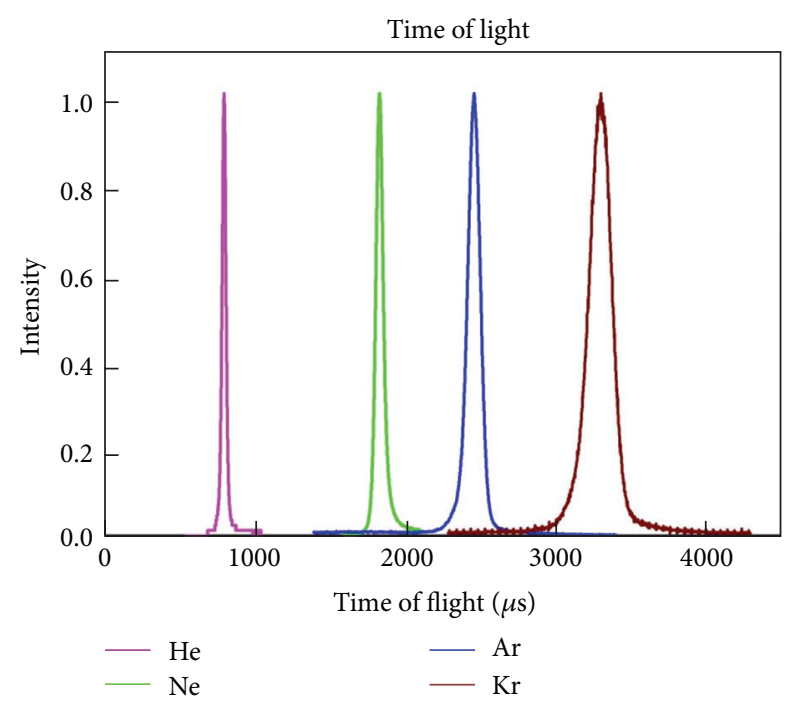

Figure 10: Time of flight for metastable atoms (1.4 m flight tube). Stagnation temperature is $300 \mathrm{~K}$ and pressure is 50 bars.

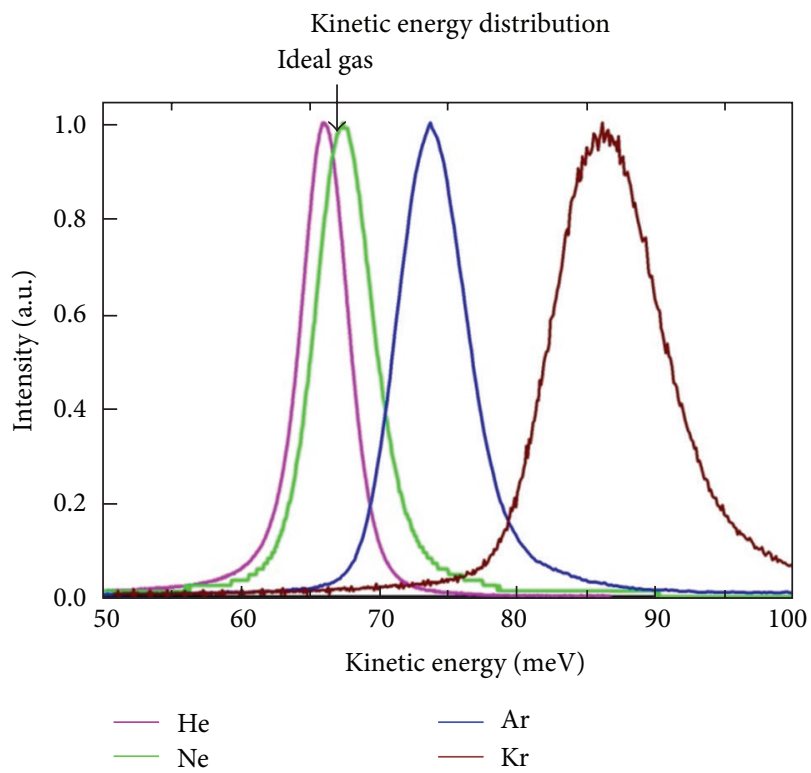

FIGURE 11: Kinetic energy distribution in the beam. Stagnation pressure and temperature as in Figure 10.

excited to neutral, metastable excited states (usually triplet states) that have long radiative lifetimes [41]. However, here we present results that were obtained by creating metastable atoms using a soft discharge mechanism inside the nozzle itself $[37,40]$. This is a more efficient mechanism that creates large numbers of excited metastable atoms in the beam. The metastable atoms created by this mechanism contain sufficient electronic energy $(10-20 \mathrm{eV})$ to allow them to be readily detected on a microchannel plate electron multiplier.

Figure 10 displays the pulse arrival time at a distance of $1 \mathrm{~m}$ downstream from the nozzle for expansions of the various rare gases. From these distributions, the average beam

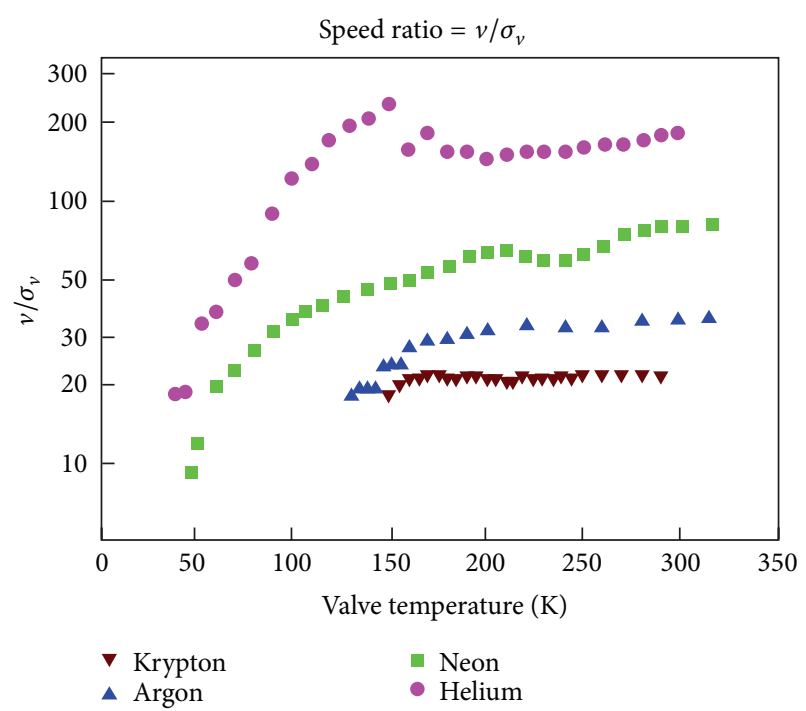

(a)

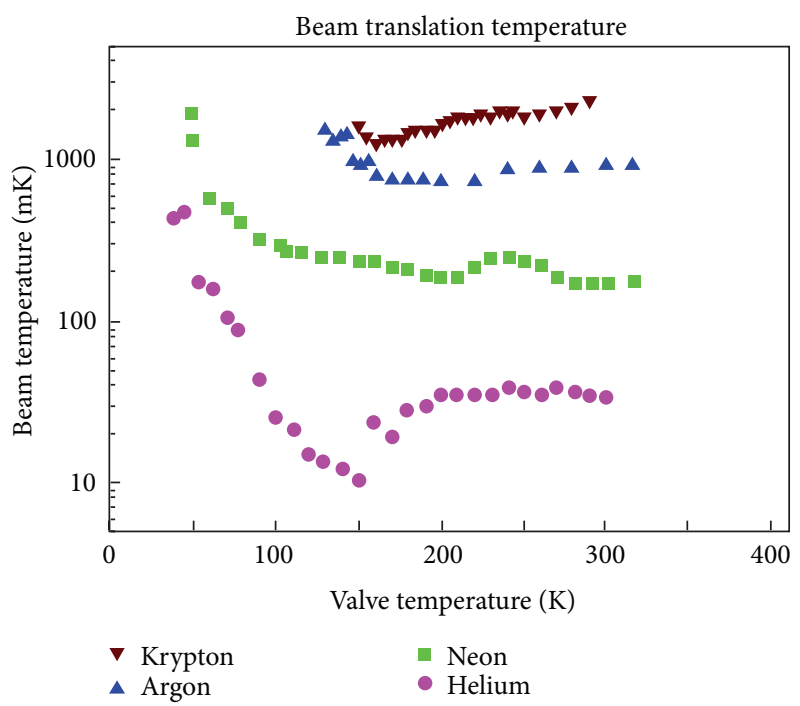

(b)

FIGURE 12: Speed ratio and attained temperature for various gases. Stagnation pressure is as in Figure 10. Errors in the measurements are $10 \%$.

velocity, $v$, and its standard deviation, $\sigma_{v}$, may be calculated. From these parameters, we can calculate the speed ratio, $S$ :

$$
S=\frac{v}{\sigma_{v}}
$$

The speed ratio allows us to calculate the translational temperature assuming an ideal gas adiabatic expansion. It is evident from Figure 10 that helium produces the coldest beam and that the temperature deteriorates as the mass of the rare gas is increased. It is illuminating to compare the experimental results with those calculated for an ideal gas expansion. This is displayed in Figure 11, where we have replotted the data of Figure 10 after converting the time-offlight distributions into kinetic energy distributions. 


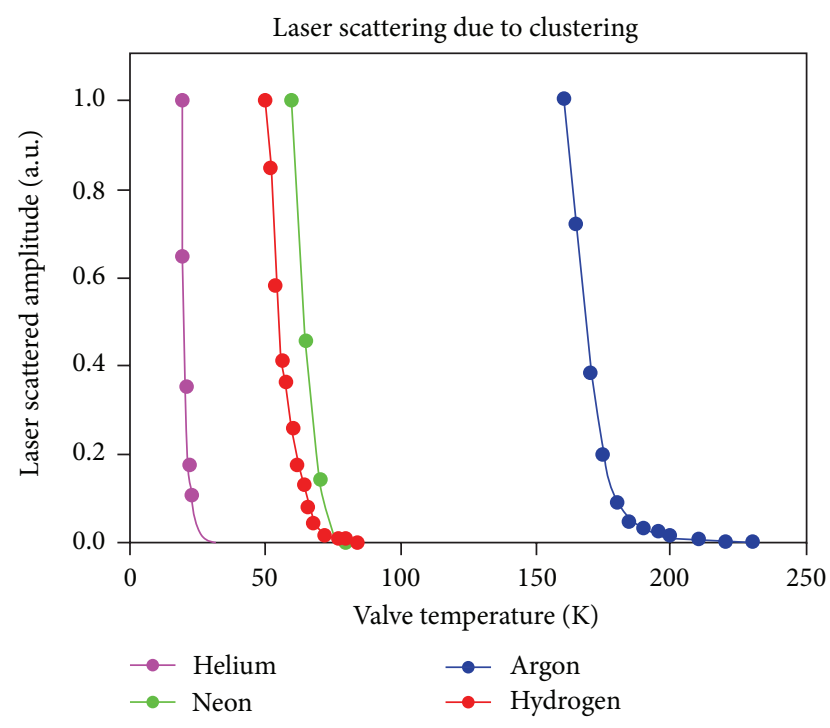

FIGURE 13: Scattered laser light, indicating extensive cluster growth below specific temperatures for each expansion gas.

As can be seen in Figure 11, only helium and neon give kinetic energy distributions that are close to the calculated value for a monatomic ideal gas. Argon and even more so krypton deviate significantly from the results expected for an ideal monatomic gas. This is undoubtedly due to the formation of rare gas clusters for these more highly polarizable gases. It is the formation of clusters during the expansion, along with the consequent release of the heat of condensation that limits the cooling of the expanding jet [18]. This phenomenon can be better seen if we plot the velocity ratio, $S$, or the corresponding translational temperature, as the temperature of the nozzle is lowered (Figure 12).

Figure 12 indicates that a high speed ratio (and a low beam temperature) can be attained readily for helium and neon, with progressively worsening conditions for argon and krypton, due to their tendency to form clusters because of their higher polarizability. Extensive clustering in the molecular beam can be detected by the enhanced light scattering that occurs by clusters, as opposed to rare gas atoms. To investigate this effect, we measured the scattered light that is detected when the focused 4 th harmonic radiation of a Nd-YAG laser $(266 \mathrm{~nm}, 2 \mathrm{~ns}$ pulse duration) is used to irradiate the expanding gas near the nozzle. The experiment was conducted using $\mathrm{He}, \mathrm{Ne}, \mathrm{H}_{2}$, and $\mathrm{Ar}$ as the expansion gas, as a function of the nozzle temperature. As displayed in Figure 13, it was found that for each gas there is a critical nozzle temperature below which clusters readily form.

We have shown that low temperatures (as low as $10 \mathrm{mK}$ ) can be achieved for pure helium expansions and $200 \mathrm{mK}$ for neon, the question now is what limits can be achieved when we seed the beam with heavier molecules at low concentration? Obviously the limiting temperature in this case will be higher, mainly because even helium can condense on the polarizable molecules and raise the temperature by releasing the heat of condensation $[19,42]$. Using LIF (Figure 14) for known rotational constants of some molecules

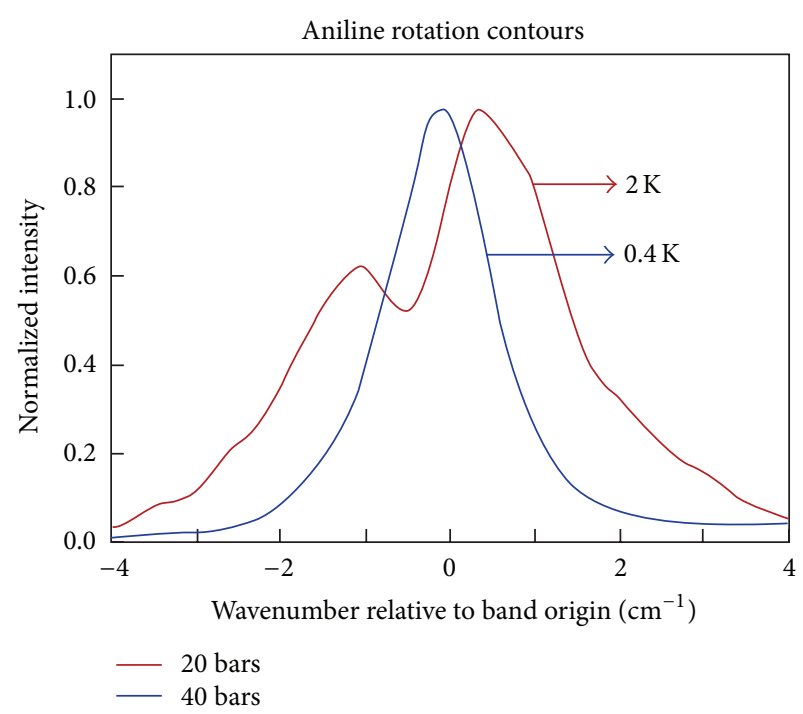

(a)

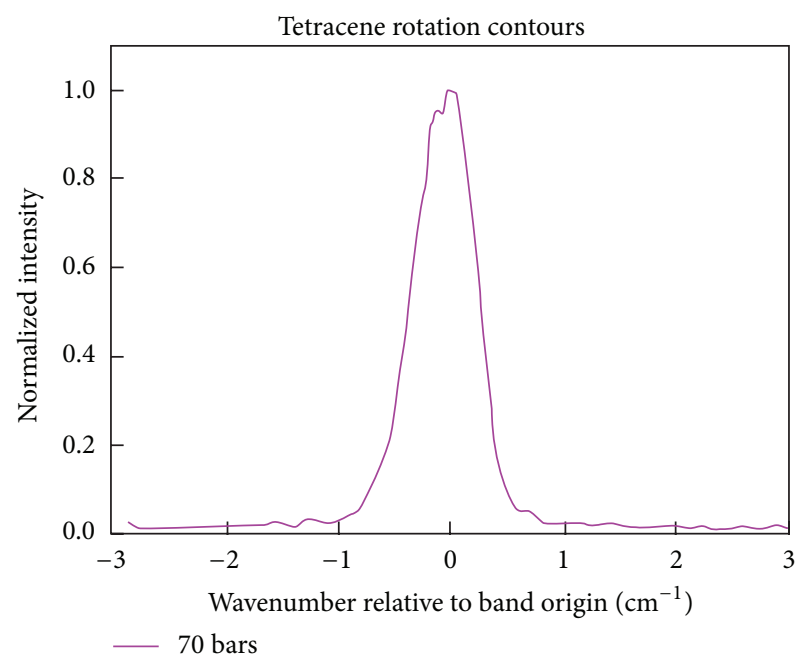

(b)

FIGURE 14: Measured rotational contour of the band origin in aniline and tetracene. Comparing the rotational contours with calculation for aniline we can estimate the rotation temperature as indicated.

we can measure the rotational temperature quite accurately [19] and it does follow the translation temperatures closely [18]. Similar conclusions can be derived from laser alignment experiments [43-45] on seeded beams. Using the data from these experiments we conclude that temperatures as low as $0.4-0.6 \mathrm{~K}$ can be achieved, even for large molecules (containing 10-50 atoms), using helium or neon high pressure sources.

\section{Skimmer Design}

Because high pressure shaped nozzles can produce high onaxis beam intensities, it is not surprising that modifications of the accepted skimmer design are required. Since the early days of low intensity continuous beams, skimmers have been 


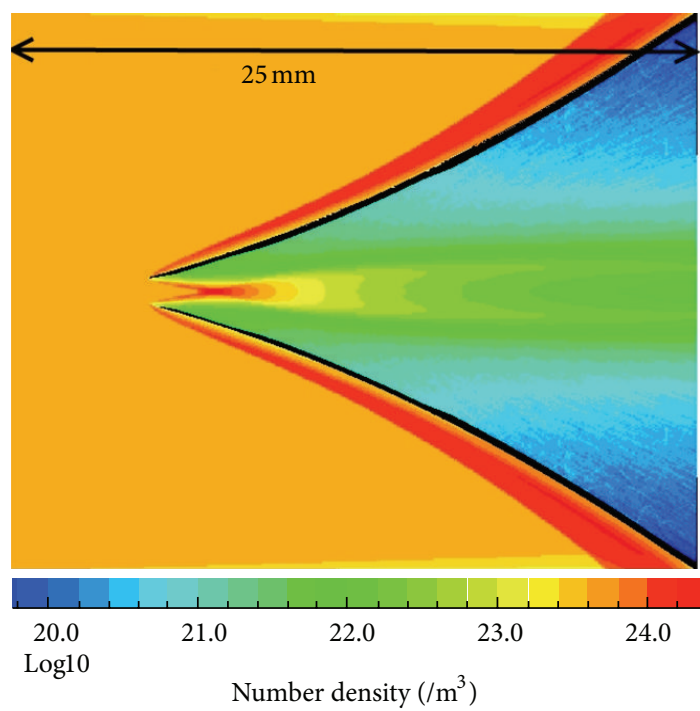

(a)

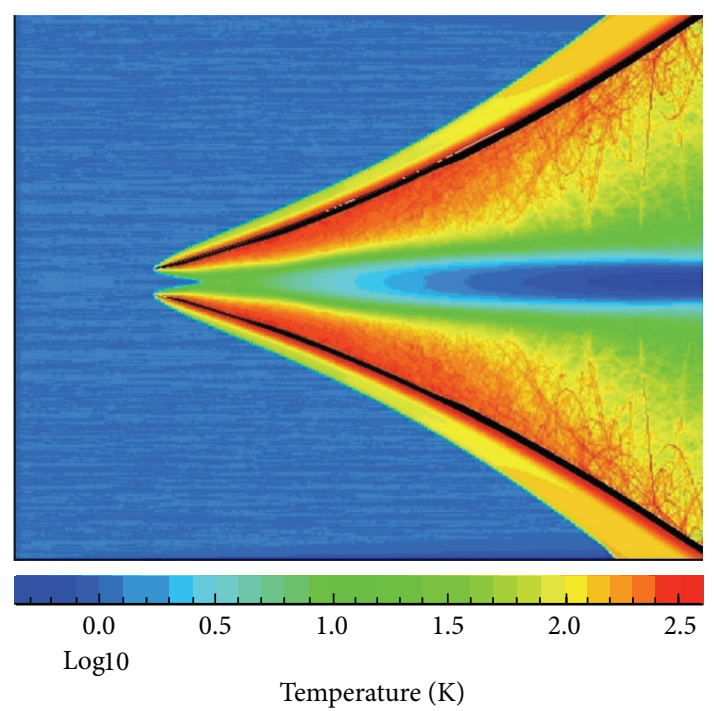

(b)

FIGURE 15: Density and temperature maps for a clogged skimmer flow at relatively high beam number density of $10^{23}$ atoms $/ \mathrm{m}^{3}$.

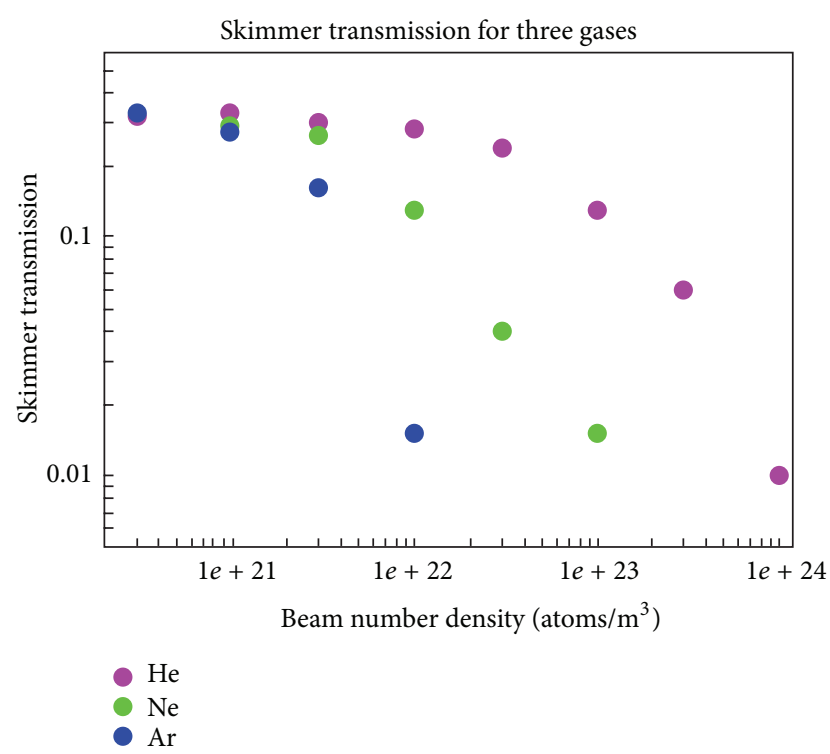

FIGURE 16: Skimmer transmission as a function of incoming beam gas density, for the skimmer depicted in Figure 15.

recognized as more than a simple bystander that extracts the central portion of the beam. The effects of molecules scattering from the finite skimmer edges have long been recognized [22, 24, 31, 46-48], and the reduced transmission of the beam through the skimmer was termed "skimmer interference." At times the term "skimmer clogging" was used to describe the dramatic reduction in skimmer transmission. To study these effects, we employed the same DSMC gas flow simulation program [25] which is eminently suitable for the gas density encountered at the skimmer. Here we show how the skimmer distance, skimmer opening, and skimmer edge

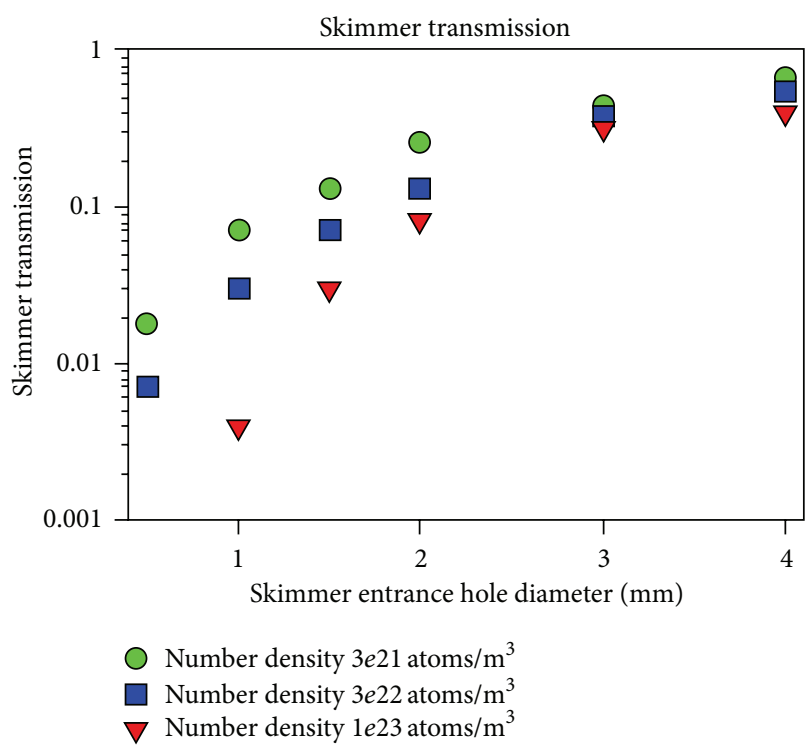

FIGURE 17: Skimmer transmission as a function of skimmer aperture (diameter) and beam density [40]. Simulations are for argon, where full energy accommodation is assumed.

sharpness influence the transmission of the beam through the skimmer.

We begin by examining a popular skimmer shape that was used extensively in low intensity continuous beams [49]. The skimmer opening is $1 \mathrm{~mm}$. and its length is $22 \mathrm{~mm}$. The included full angle at its tip is $25^{\circ}$ and at its base $70^{\circ}$. The tip sharpness is determined by the simulation mesh size and is $\sim 5 \mathrm{~mm}$. The simulation represents the flow of a collimated and cold stream of neon at $1 \mathrm{~K}$ moving at $750 \mathrm{~m} / \mathrm{s}$ to the right. We chose the neon beam number density $(3 *$ $10^{23}$ atoms $/ \mathrm{m}^{3}$ ) to represent the density of the gas from a 


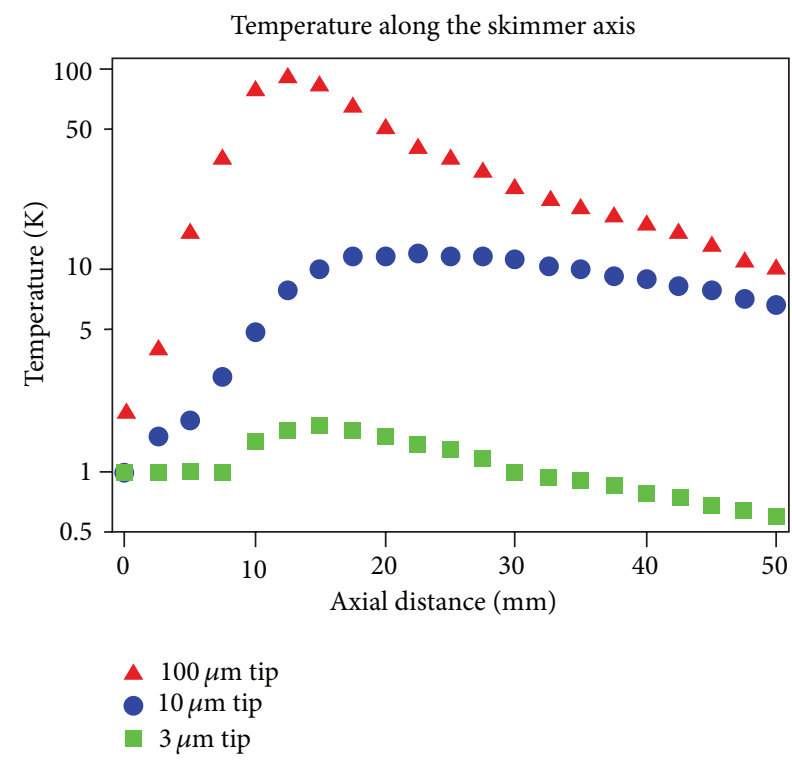

Figure 18: Temperature rise in a blunt skimmer [40]. The flowing gas is neon (energy accommodation coefficient 0.8 ) at $1 \mathrm{~K}$ and a number density of $10^{22}$ atoms $/ \mathrm{m}^{3}$. Skimmer entrance aperture is $1 \mathrm{~mm}$. The temperature soak, lasting only few $\mathrm{mS}$ can destroy fragile clusters in the beam.

$0.2 \mathrm{~mm}$. conical nozzle at a stagnation pressure of 30 bars and at a distance of $10 \mathrm{~mm}$ from the nozzle (extrapolated from Figure 3). Figure 15 represents a clogged skimmer condition with a high density gas plug formed at the skimmer throat, serving as a secondary expansion point from this higher temperature plug $(25 \mathrm{~K})$. The transmitted beam intensity at the skimmer base is less than $2 \%$ of the incoming beam number density.

Skimmer clogging and therefore low beam transmission can occur quite suddenly as the gas beam density rises. This effect is illustrated in Figure 16, where skimmer transmission as a function of beam gas density is plotted for the skimmer described above and illustrated in Figure 16. In Figure 17, skimmer transmission is defined as the ratio of the gas density at the skimmer outlet to the density at the skimmer inlet. The simulation results are sensitive to the interaction parameters of the gas with the skimmer surface (energy accommodation occurring in the gas surface collision). The accommodation coefficient rises as the mass of the gas atoms approaches that of the surface atoms (nickel). We chose accommodation coefficients of 0.4 for $\mathrm{He}, 0.8$ for $\mathrm{Ne}$, and 1 (complete accommodation) for Ar [29].

A surprising result is that in order to allow for a reasonable beam transmission one has to move the skimmer to a larger distance from the nozzle, as large as $150 \mathrm{~mm}$. (or 750 nozzle diameters!) for $\mathrm{Ne}$ or $55 \mathrm{~mm}$. for helium. Lower beam transmission is found for Ar.

The next issue we address is the how does the skimmer entrance hole affect its transmission. The simulations show that smaller hole skimmer tends to clog up at lower beam densities than a larger entrance hole skimmers.
To achieve the best transmission results, it is necessary to employ a large diameter entrance aperture $(2-4 \mathrm{~mm})$ and to move the skimmer by as much as 1000 nozzle diameters downstream from the nozzle $(100-200 \mathrm{~mm})$ to avoid strong beam attenuation in the skimmer. This is illustrated in Figure 17, where the skimmer transmission is plotted as a function of skimmer entrance diameter for conical skimmers ( $20^{\circ}$ full opening angle) subjected to gas densities ranging from $3 \times 10^{21}$ to $1 \times 10^{23}$ atoms $/ \mathrm{m}^{3}$. The necessity of using narrow conical skimmers with large diameter entrance apertures, positioned far downstream, is a very unorthodox result that will require the redesign of source chambers for a large number of currently employed high intensity supersonic sources.

The last question we wish to address is how sharp does the skimmer edge need to be? We can simulate the temperature rise of the cold beam due to molecules scattering from the room temperature skimmer edges. The results are shown in Figure 18. A long conical skimmer was used and the axial temperature along its axis is shown. Beam number density is $10^{22}$ of helium. A wall thickness of $3 \mu \mathrm{m}$ causes little beam heating in the skimmer. A $10 \mu \mathrm{m}$ tip thickness is already causing significant beam heating, and a $100 \mu \mathrm{m}$ wall is simply bad. Sharply honed or electropolished edges can be machined on the skimmer tip to an edge that is smaller than 3 microns.

\section{Summary and Conclusions}

We have tested by simulation and experiments the flow characteristic of several nozzle shapes. A shaped nozzle can increase dramatically the on-axis beam number density at a given distance from the nozzle, while achieving high speed ratio. Our simulations do not include a clustering mechanism; thus we attribute the limited cooling achieved in experiments to cluster formation in the expanding jet. This is corroborated by following the cluster formation in the beam by laser light scattering. The trends in cluster formation below a certain source temperature (for each specific gas) support this assumption in general, but we could not follow this quantitatively. We tested the behavior of a popular skimmer shape to the increased gas load achieved in shaped nozzle sources. A clearer picture emerges as to what is happening when skimmer clogging occurs (i.e., skimmer interference effects). We show that skimmer interference is gas dependent (accommodation coefficient that is specific for each gas) and is also a function of the skimmer aperture and its edge sharpness. When using high pressure sources with a shaped nozzle the skimmer has to be placed some 500-1000 nozzle diameters away from the source. The required modifications in vacuum system design may prevent some common errors when replacing the source type to a high pressure source with a shaped nozzle.

\section{Conflict of Interests}

The author declares that there is no conflict of interests regarding the publication of this paper. 


\section{References}

[1] R. C. Campargue, Atomic and Molecular Beams: The State of the Art 2000, Springer, Berlin, Germany, 2001.

[2] G. Scoles, Atomic and Molecular Beam Methods, Oxford University Press, Oxford, UK, 1989-1992.

[3] H. Pauly, Atom, Molecule, and Cluster Beams I, Springer, Berlin, Germany, 2000.

[4] H. Haberland, Clusters of Atoms and Molecules, Springer, Berlin, Germany, 1994.

[5] R. Campargue, A. Lebehot, and J. C. Lemonnier, in Rarefied Gas Dynamics, pp. 1033-1045, 1977.

[6] A. Amirav, U. Even, and J. Jortner, "Absorption spectroscopy of ultracold large molecules in planar supersonic expansions," Chemical Physics Letters, vol. 83, no. 1, pp. 1-4, 1981.

[7] W. R. Gentry and C. F. Giese, "Ten-microsecond pulsed molecular beam source and a fast ionization detector," Review of Scientific Instruments, vol. 49, p. 595, 1978.

[8] B. Yan, P. F. H. Claus, B. G. M. van Oorschot et al., "A new high intensity and short-pulse molecular beam valve," Review of Scientific Instruments, vol. 84, no. 2, Article ID 023102, 2013.

[9] M. D. Barry, N. P. Johnson, and P. A. Gorry, "A fast $(30 \mu \mathrm{s})$ pulsed supersonic nozzle beam source: application to the photodissociation of $\mathrm{CS}_{2}$ at $193 \mathrm{~nm}$," Journal of Physics E: Scientific Instruments, vol. 19, no. 10, pp. 815-819, 1986.

[10] D. Irimia, D. Dobrikov, R. Kortekaas et al., "A short pulse $(7 \mu \mathrm{s}$ FWHM) and high repetition rate $(\mathrm{dc}-5 \mathrm{kHz})$ cantilever piezovalve for pulsed atomic and molecular beams," Review of Scientific Instruments, vol. 80, no. 11, Article ID 113303, 2009.

[11] D. L. Proctor, D. R. Albert, and H. F. Davis, "Improved piezoelectric actuators for use in high-speed pulsed valves," Review of Scientific Instruments, vol. 81, Article ID 023106, 2010.

[12] M. R. Adriaens, W. Allison, and B. Feuerbacher, "A pulsed molecular beam source," Journal of Physics E: Scientific Instruments, vol. 14, no. 12, article no. 006, pp. 1375-1377, 1981.

[13] D. Bahat, O. Cheshnovsky, U. Even, N. Lavie, and Y. Magen, "Generation and detection of intense cluster beams," Journal of Physical Chemistry, vol. 91, no. 10, pp. 2460-2462, 1987.

[14] D. Pentlehner, R. Riechers, B. Dick et al., "Rapidly pulsed helium droplet source," Review of Scientific Instruments, vol. 80, no. 4, Article ID 043302, 2009.

[15] E. Hendell and U. Even, "Cluster-surface interaction at high kinetic energy. I. Electron emission," The Journal of Chemical Physics, vol. 103, no. 20, pp. 9045-9052, 1995.

[16] O. F. Hagena, "Pulsed valve for supersonic nozzle experiments at cryogenic temperatures," Review of Scientific Instruments, vol. 62, pp. 2038-2039, 1991.

[17] C. E. Otis and P. M. Johnson, "A simple pulsed valve for use in supersonic nozzle experiments," Review of Scientific Instruments, vol. 51, p. 1128, 1980.

[18] M. Hillenkamp, S. Keinan, and U. Even, "Condensation limited cooling in supersonic expansions," Journal of Chemical Physics, vol. 118, no. 19, pp. 8699-8705, 2003.

[19] U. Even, J. Jortner, D. Noy, N. Lavie, and C. Cossart-Magos, "Cooling of large molecules below $1 \mathrm{~K}$ and He clusters formation," Journal of Chemical Physics, vol. 112, no. 18, pp. 8068-8071, 2000.

[20] W. Christen, "Stationary flow conditions in pulsed supersonic beams," The Journal of Chemical Physics, vol. 139, Article ID 154202, 2013.
[21] G. A. Bird, Molecular Gas Dynamics and the Direct Simulation of Gas Flows, Oxford University Press, Oxford, UK, 1994.

[22] W. R. Gentry and C. F. Giese, "High-precision skimmers for supersonic molecular beams," Review of Scientific Instruments, vol. 46, no. 1, p. 104,1975 .

[23] G. A. Bird, "Transition regime behavior of supersonic beam skimmers," Physics of Fluids, vol. 19, no. 10, pp. 1486-1491, 1976.

[24] J. Braun, P. K. Day, J. P. Toennies, G. Witte, and E. Neher, "Micrometer-sized nozzles and skimmers for the production of supersonic He atom beams," Review of Scientific Instruments, vol. 68, no. 8, pp. 3001-3009, 1997.

[25] G. Bird, http://www.gab.com.au/.

[26] K. J. Daun, G. J. Smallwood, and F. Liu, "Molecular dynamics simulations of translational thermal accommodation coefficients for time-resolved LII," Applied Physics B, vol. 94, no. 1, pp. 39-49, 2009.

[27] W. F. N. Santos, "Gas-surface interaction effect on round leading edge aerothermodynamics," Brazilian Journal of Physics, vol. 37, no. 2, pp. 337-348, 2007.

[28] J. F. Padilla and I. D. Boyd, "Assessment of gas-surface interaction models for computation of rarefied hypersonic flow," Journal of Thermophysics and Heat Transfer, vol. 23, no. 1, pp. 96-105, 2009.

[29] D. J. Rader, W. M. Trott, J. R. Torczynski, J. N. Castañeda, and T. W. Grasser, Sandia National Laboratories SAND20056084, 2005, http://prod.sandia.gov/techlib/access-control.cgi/ 2005/056084.pdf.

[30] H. Ashkenas and S. F. Sherman, Proceedings of the 4th International Symposium on Rarefied Gas Dynamics: Experimental Methods in Rarefied Gas Dynamics, Academic Press, New York, NY, USA, 1966.

[31] R. Campargue, "Characteristics of supersonic beams applicable in collision or semi-collision experiments," Journal de Chimie Physique et de Physico-Chimie Biologique, vol. 77, no. 3, p. R15, 1980.

[32] R. Campargue, "Progress in overexpanded supersonic jets and skimmed molecular beams in free-jet zones of silence," The Journal of Physical Chemistry, vol. 88, no. 20, pp. 4466-4414, 1984.

[33] S. Semushin and V. Malka, "High density gas jet nozzle design for laser target production," Review of Scientific Instruments, vol. 72, no. 7, pp. 2961-2965, 2001.

[34] H. V. Tafreshi, G. Benedek, P. Piseri, S. Vinati, E. Barborini, and P. Milani, "A simple nozzle configuration for the production of low divergence supersonic cluster beam by aerodynamic focusing," Aerosol Science and Technology, vol. 36, no. 5, pp. 593606, 2002.

[35] J. P. Toennies and K. Winkelman, "Theoretical studies of highly expanded free jets: Influence of quantum effects and a realistic intermolecular potential," The Journal of Chemical Physics, vol. 66, no. 9, p. 3965, 1977.

[36] J. T. McDaniels, R. E. Continetti, and D. R. Miller, "The effect of nozzle geometry on cluster formation in molecular beam sources," in Proceedings of the 23rd international Symposium on Rarefied Gas Dynamics, A. D. Ketsdever, Ed., American Institute of Physics, 2003.

[37] K. Luria, N. Lavie, and U. Even, "Dielectric barrier discharge source for supersonic beams," Review of Scientific Instruments, vol. 80, Article ID 104102, 2009.

[38] E. Narevicius, C. G. Parthey, A. Libson et al., "An atomic coilgun: Using pulsed magnetic fields to slow a supersonic beam," New Journal of Physics, vol. 9, article 358, 2007. 
[39] E. Narevicius, A. Libson, M. F. Riedel et al., "Coherent slowing of a supersonic beam with an atomic paddle," Physical Review Letters, vol. 98, no. 10, Article ID 103201, 2007.

[40] K. Luria, W. Christen, and U. Even, "Generation and propagation of intense supersonic beams," Journal of Physical Chemistry A, vol. 115, no. 25, pp. 7362-7367, 2011.

[41] W. Christen, "Stationary flow conditions in pulsed supersonic beams," The Journal of Chemical Physics, vol. 139, no. 15, Article ID 154202, 2013.

[42] U. Even, I. Al-Hroub, and J. Jortner, "Small he clusters with aromatic molecules," Journal of Chemical Physics, vol. 115, no. 5, pp. 2069-2073, 2001.

[43] H. Stapelfeldt, "Laser aligned molecules: applications in physics and chemistry," Physica Scripta, vol. T110, p. 132, 2004.

[44] H. Stapelfeldt and T. Seideman, "Colloquium: Aligning molecules with strong laser pulses," Reviews of Modern Physics, vol. 75, no. 2, pp. 543-557, 2003.

[45] I. Nevo, L. Holmegaard, J. H. Nielsen et al., "Laser-induced 3D alignment and orientation of quantum state-selected molecules," Physical Chemistry Chemical Physics, vol. 11, no. 42, pp. 9912-9918, 2009.

[46] N. F. Ramsey, Molecular Beams, Oxford University Press, Oxford, UK, 1956.

[47] A. B. Bailey, R. Dawbarn, and M. R. Busby, "Effects of skimmer and endwall temperature of condensed molecular beams," AIAA Journal, vol. 14, no. 1, pp. 91-92, 1976.

[48] D. C. Jordan, R. Barling, and R. B. Doak, "Refractory graphite skimmers for supersonic free-jet, supersonic arc-jet, and plasma discharge applications," Review of Scientific Instruments, vol. 70, no. 3, pp. 1640-1648, 1999.

[49] Beam-Dynamics, http://www.beamdynamicsinc.com/skimmer specs.htm. 

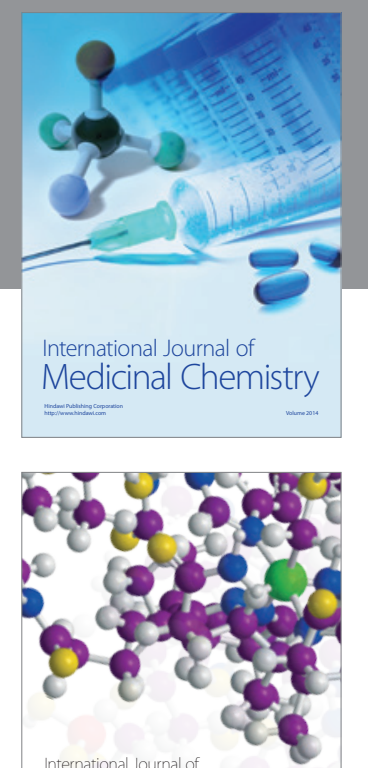

\section{Carbohydrate} Chemistry

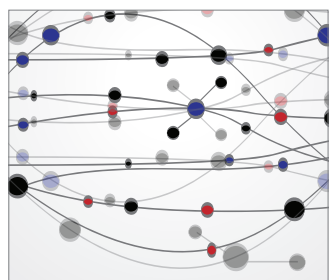

The Scientific World Journal
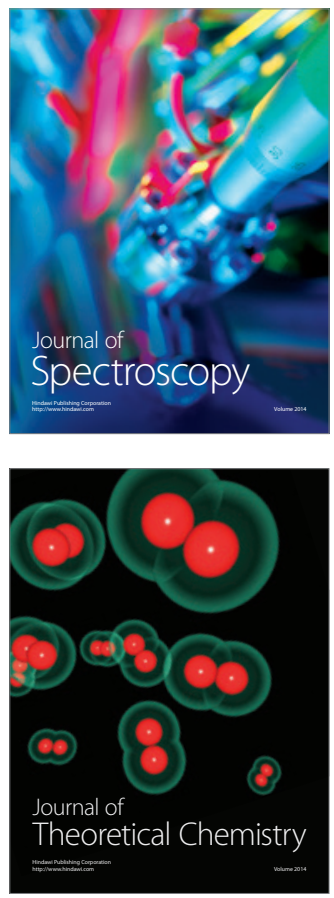
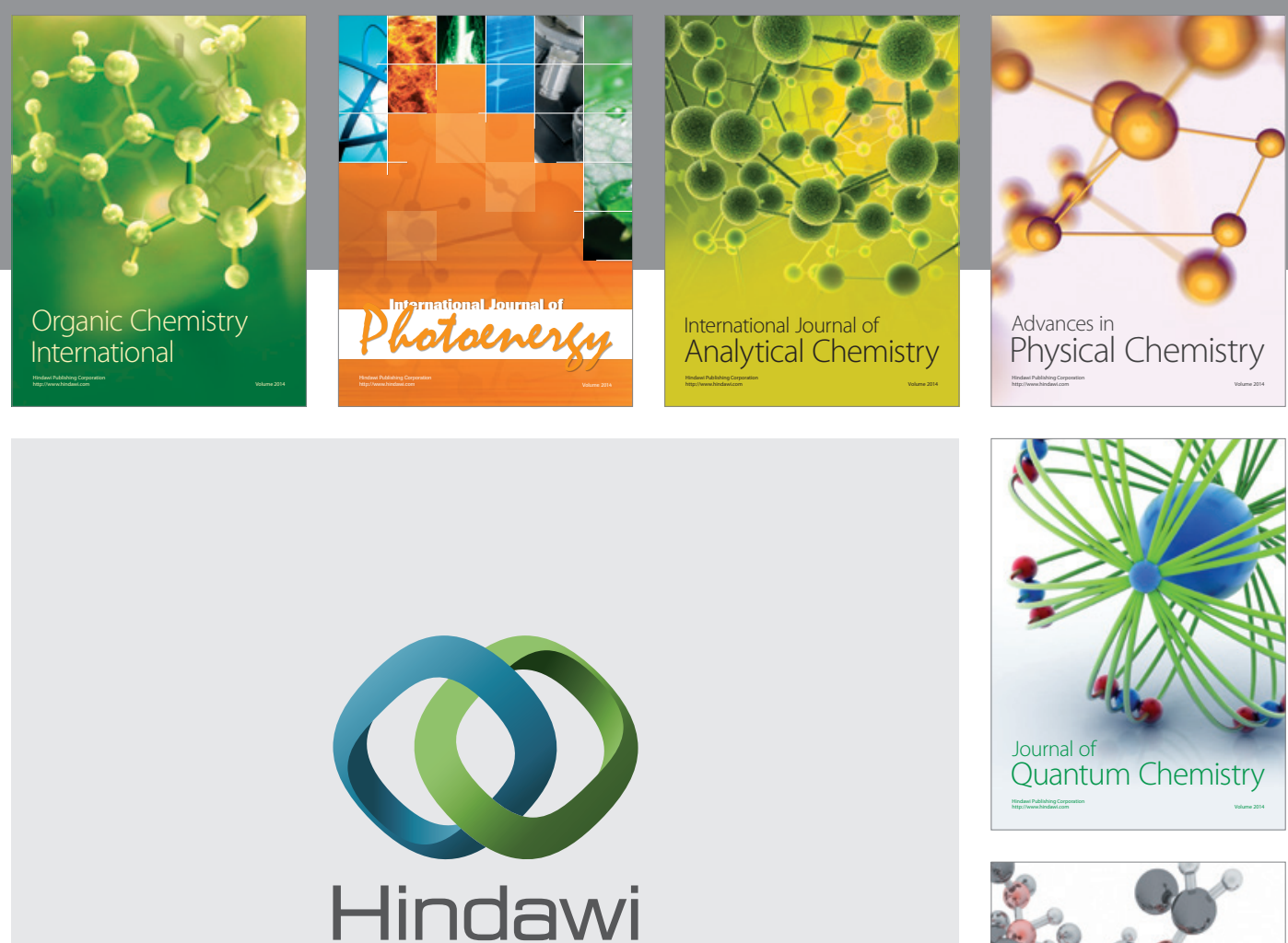

Submit your manuscripts at

http://www.hindawi.com

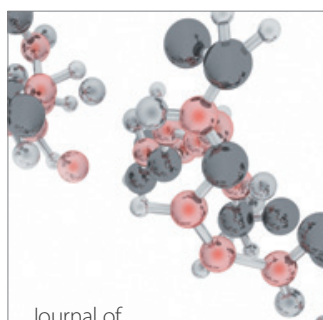

Analytical Methods

in Chemistry

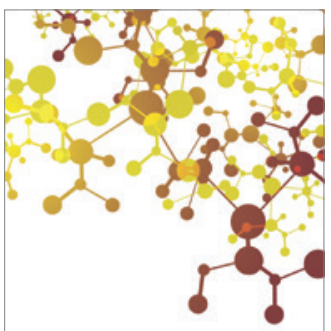

Journal of

Applied Chemistry

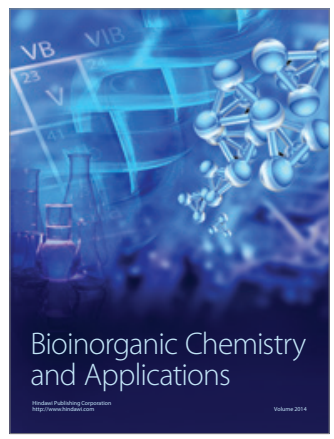

Inorganic Chemistry
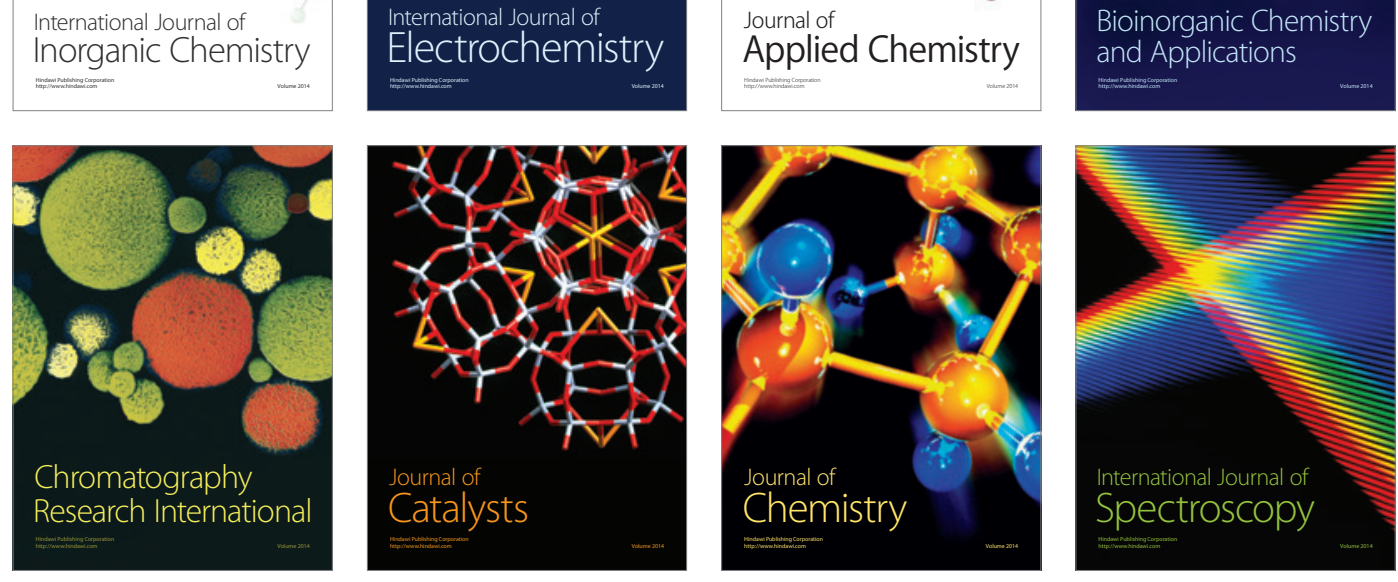